\title{
Case Report: Identification of an HNF1B p.Arg527Gln mutation in a Maltese patient with atypical early onset diabetes and diabetic nephropathy
}

\author{
Nikolai Paul Pace ${ }^{1 *}$, Johann Craus $^{2}$, Alex Felice ${ }^{1}$ and Josanne Vassallo ${ }^{3}$
}

\begin{abstract}
Background: The diagnosis of atypical non-autoimmune forms of diabetes mellitus, such as maturity onset diabetes of the young (MODY) presents several challenges, in view of the extensive clinical and genetic heterogeneity of the disease. In this report we describe a case of atypical non autoimmune diabetes associated with a damaging HNF1 $\beta$ mutation. This is distinguished by a number of uncharacteristic clinical features, including early-onset obesity, the absence of renal cysts and diabetic nephropathy. HNF1ß-MODY (MODY5) is an uncommon form of monogenic diabetes that is often complicated by a wide array of congenital morphological anomalies of the urinary tract, including renal cysts. This report expands on the clinical phenotypes that have been described in the context of HNF1 $\beta$ mutations, and is relevant as only isolated cases of diabetic nephropathy in the setting of MODY5 have been reported.
\end{abstract}

Case presentation: An obese Maltese female with non-autoimmune diabetes, microalbuminuria, glomerular hyperfiltration, fatty liver and no renal cysts was studied by whole exome sequencing to investigate potential genes responsible for the proband's phenotype. A rare missense mutation at a highly conserved site in exon 8 of HNF1 $\beta$ was identified (c.1580G > A, NM_000458.3, p.Arg527Gln), with multiple in-silico predictions consistent with pathogenicity. This mutation has not been previously characterised. Additionally, several common susceptibility variants associated with early-onset obesity, polygenic type 2 diabetes and nephropathy were identified in the proband that could impose additional effects on the phenotype, its severity or its clinical course.

Conclusion: This report highlights several atypical features in a proband with atypical diabetes associated with an HNF1 $\beta$ missense mutation. It also reinforces the concept that monogenic causes of diabetes could be significant contributors to disease burden in obese individuals with atypical diabetes.

Keywords: MODY 5, Diabetic nephropathy, Obesity, HNF1 $\beta$, Atypical diabetes

\section{Background}

The diagnosis of atypical non-autoimmune diabetes presents several challenges. These forms of diabetes are best exemplified by maturity onset diabetes of the young (MODY). MODY is a rare group of genetically heterogenous conditions characterised by beta cell dysfunction and defects in insulin secretion. Systematic screening of European and

\footnotetext{
* Correspondence: nikolai.p.pace@um.edu.mt

${ }^{1}$ Centre for Molecular Medicine and Biobanking, University of Malta, Msida, Malta

Full list of author information is available at the end of the article
}

North American paediatric populations has identified a prevalence range of 1.2 to $4.2 \%$ [1-3]. Population-specific differences in the prevalence of MODY have also been reported $[4,5]$.

A wide array of phenotypic heterogeneity is observed in subjects with different mutations in the various genes implicated in the pathogenesis of monogenic diabetes, as this disease can mimic either type 1 or type 2 diabetes mellitus. Correctly making the diagnosis of MODY is essential in view of the therapeutic and prognostic implications. Furthermore, the autosomal dominant pattern

(c) The Author(s). 2018 Open Access This article is distributed under the terms of the Creative Commons Attribution 4.0 International License (http://creativecommons.org/licenses/by/4.0/) which permits unrestricted use, distribution, and reproduction in any medium, provided you give appropriate credit to the original author(s) and the source, provide a link to the Creative Commons license, and indicate if changes were made. The Creative Commons Public Domain Dedication waiver (http://creativecommons.org/publicdomain/zero/1.0/) applies to the data made available in this article, unless otherwise stated. 
of inheritance mandates genetic counselling and family follow-up. The increasing availability of whole exome or targeted capture followed by high throughput sequencing facilitates the molecular diagnosis of monogenic diabetes, particularly in cases where the clinical phenotype is atypical or complicated by clinical features that are not routinely associated with MODY. Increasingly, next generation sequencing followed by interpretation using publicly-available aggregate exome variant datasets offers an unrivalled scope for the discovery and annotation of novel variants.

Identifying patients with monogenic diabetes requires an index of clinical suspicion of the disease followed by molecular diagnosis to confirm the underlying genetic defect. Guidelines issued by the International Society for Paediatric and Adolescent Diabetes (ISPAD) are available to advise clinicians, and genetic diagnosis is available in many healthcare systems [6] . Despite these guidelines, studies have shown that genetic testing for MODY is under requested and clinical prediction models have been developed to aid the identification of likely candidates for molecular genetic testing $[7,8]$.

In this report, we describe a rare damaging missense mutation in HNF1 $\beta$ that was identified in a proband with early-onset atypical diabetes with no morphological renal tract anomalies. The proband had glomerular hyperfiltration and early stage diabetic nephropathy, which is unusual in the setting of MODY5. Because of the unusual features of nephropathy in the absence of renal cysts and obesity, we performed whole exome sequencing of the proband.

\section{Methods}

\section{Patient}

This study was approved by the ethics institutional review board of the University of Malta (IRB 71/2013). Written informed consent was obtained from the proband. The mother had demised and the proband's brother and father were not available for genetic analysis at the time of the study.

\section{Sample preparation and whole exome sequencing}

Genomic DNA was extracted from a peripheral blood sample taken from the proband using a QIAamp DNA extraction kit (Qiagen, Hilden, Germany), and checked for purity and integrity using agarose gel electrophoresis and UV spectrophotometry. Sample preparation and exon enrichment for next generation sequencing was performed using a SureSelectXT All Exon V5 kit (Agilent Technologies, Santa Clara, CA). $3 \mu \mathrm{g}$ of DNA was processed according to manufacturer's instructions. Paired end sequencing was carried out on an Illumina HiSeq 2500 sequencer (Illumina, Inc., San Diego, CA, USA).

\section{Exome sequencing alignment, variant calling and mutation detection}

Image analysis was performed with the default parameters of Illumina RTA pipeline, and base calling was carried out using CASAVA. The sequence reads were mapped and aligned to the Human Reference Genome (UCSC hg19, NCBI build 37) using the BurrowsWheeler transformation algorithm, and duplicated reads were removed using Picard $[9,10]$. FastQC was used to check the quality of sequence data [11]. Calling of SNPs and InDels was done using GATK Unified Genotyper, which uses a Bayesian genotype likelihood model to report alleles and Phred-scaled confidence values [12]. Variants (SNVs and indels) were called with SAMTools, with reference to public databases including dbSNP and 1000 Genomes and gnomAD [13]. Analysis was performed with preference to variants located in genes implicated in atypical non-autoimmune forms of diabetes and early-onset obesity. The prioritized candidate gene list was obtained by reviewing publications in PubMed and OMIM. Analysis focused on non-synonymous coding variants, frameshift indels, and variants affecting splice sites, as these are most likely to be pathogenic. Non-exonic and synonymous variants were excluded from further analysis. Missense variants were evaluated for functional impact using a variety of in-silico prediction tools including SIFT [14], Polyphen2 [15], MelaLR [16], MetaSVN [16], fathmm-MKL [17], DANN [18], CADD [19], MutationTaster [20], Mutation Assesser [21] and LRT [22].

\section{Sanger sequencing}

Sanger sequencing using standard PCR amplification procedures was carried out to confirm the selected candidate variants of interest in the proband and in 300 unrelated controls of Maltese ethnicity. A 242 base pair region in exon 8 of HNF1 $\beta$ was amplified using the following forward primer (GGG CTC TGT ACC TGT GTC TT) and reverse primer (CCA TGG CCT TAT CAC ACC CT) with an annealing temperature of 54 degrees.

\section{Case presentation}

\section{Clinical features}

The proband is a 25 year old Caucasian female born from nonconsanguineous parents of Maltese ethnicity. She developed obesity in early childhood, with a body weight at the 97th centile at the age of 9 years. She was diagnosed with diabetes mellitus at age 11 following her presentation with osmotic symptoms of hyperglycaemia. No diabetic ketoacidosis at diagnosis was present, and both glutamic acid decarboxylase and islet cell antibodies were negative. She was initially treated by diet and lifestyle changes, and eventually started on metformin during childhood. 
The proband became pregnant at age 21 years, and she delivered a healthy but macrosomic male infant by Caesarean section at 35 weeks of gestation weighing 5 . $18 \mathrm{~kg}$. Her glycaemic control deteriorated significantly during pregnancy and was managed by combination treatment of isophane and soluble insulin. Significant weight gain also developed during pregnancy, with a BMI up to $37 \mathrm{~kg} / \mathrm{m}^{2}$. Pre-proliferative diabetic retinopathy was also present in the proband.

Since pregnancy the proband developed persistent and significant microalbuminuria (urine microalbumin $>400 \mathrm{mg} / \mathrm{L}$ ), leading to macroalbuminuria (albumincreatinine ratio $>3000 \mathrm{mg} / \mathrm{g}$ ) and glomerular hyperfiltration (eGFR $>170 \mathrm{mls} / \mathrm{min} / 1.73 \mathrm{~m}^{2}$ ) with normal creatinine levels. Urinalysis and urine microscopy showed no significant findings. Ultra-sonographic examination of the abdomen revealed normal size and echotexture in both kidneys, without any signs of obstructive uropathy, and normal cortical thickness and preservation of cortico-medullary differentiation. No evidence of autoimmune nephropathy or glomerulonephritis was present, with normal ANA, ANCA, C3, $\mathrm{C} 4$, rheumatoid factor IgM, uric acid, C-reactive protein and serum immunoglobulin levels. The proband also developed deranged liver function tests, with moderately elevated gamma glutamyl transferase and alanine transaminase levels. A viral hepatitis screen was negative, and hepatomegaly with no focal lesions and changes of a fatty liver were also evident on abdominal ultrasound.

As an adult, she is presently overweight (BMI $28 \mathrm{~kg} / \mathrm{m}^{2}$ ) and glycaemic control is achieved by a combination of oral hypoglycaemic agents including metformin $1 \mathrm{~g}$ tds, gliclazide $80 \mathrm{mg}$ tds and vildagliptin $50 \mathrm{mg}$ daily. The proband however shows poor glycaemic control on combination oral treatment, with $\mathrm{HbA} 1 \mathrm{c}$ values around $10 \%$. Her fasting C-peptide concentration at the time of referral for genetic analysis was $1.4 \mathrm{ng} / \mathrm{mL}$, indicating endogenous insulin production. Her HOMA-IR at the time was 2.8 (fasting insulin $5.7 \mathrm{mIU} / \mathrm{L}$, fasting blood glucose $11.05 \mathrm{mmol} / \mathrm{l})$. A repeat fasting $\mathrm{C}$-peptide decreased to $1.1 \mathrm{ng} / \mathrm{mL}$ within 1 year. In addition, the proband was started on an angiotensin-converting enzyme inhibitor (perindopril, $8 \mathrm{mg} /$ day) for reno-protection in view of the proteinuria and gradual increase in blood pressure that developed in the post pregnancy period. Her blood pressure control is generally well controlled on perindopril.

The proband's mother had developed diabetes at age 23 , and was treated with oral hypoglycaemic agents and eventually insulin. The father developed type 2 diabetes aged 68 years, and the proband's brother developed diabetes aged 36 years. In addition, three maternal aunts also had a history of diabetes mellitus. An overview of the pedigree is shown in Fig. 1 .
In view of the above clinical picture, an initial diagnosis of early-onset type 2 diabetes complicated by fatty liver and diabetic nephropathy was made. The case was subsequently revised when the proband was offered and consented to genetic analysis for monogenic diabetes. In keeping with the strong family history of early-onset diabetes and the absence of ketoacidosis, the monogenic diabetes probability calculator showed a high probability of MODY (> 75.5\% positive predictive value) [8]. Whole exome sequencing was the analytical method of choice, given the possibility of a primary genetic defect underlying the associated obesity, which is an unusual feature of monogenic diabetes.

\section{Mutation detection}

A summary of the mapped sequencing data is shown in Table 1. WES yielded a total of 118,986 variants, of which 19,598 were classified as silent mutations, 16,869 as missense variants and 128 as nonsense mutations. Variants were filtered according to their frequency, location, functional consequences and clinical phenotype as outlined in the methods section.

No nonsense, frameshift, in-frame indels and variants affecting splicing sites were detected in the proband in any of the common MODY genes. A heterozygous missense mutation with predicted damaging effects in HNF1 $\beta$ was identified in the proband - chromosome 17, position 36,059,155, c.1580G > A, NM_000458.3, p. Arg527Gln, exon 8. In-silico analysis of pathogenicity using various bioinformatic approaches are shown in Table 2. Clearly, multiple lines of computational evidence provide support for a deleterious effect of this mutation. Evolutionary conservation analysis also shows that the p.Arg527Gln mutation occurs at a highlyconserved position within the protein sequence, and that this residue is highly conserved across multiple species. The mutation was confirmed by Sanger sequencing in the proband (Fig. 2). In addition, the mutation was not detected by Sanger sequencing in 300 DNA samples of Maltese ethnicity. In the Genome Aggregation database (gnomAD), which reports summary data from largescale exome and genome sequencing projects, the HNF1 $\beta$ p.Arg527Gln mutation has a very low frequency $(3 / 246266$, frequency $=0.00001218)$. This mutation lies in the C-terminal transactivation domain of the protein, and is predicted to be likely pathogenic based on the above criteria.

In addition to mutations in known genes implicated in monogenic diabetes, we also screened sequence data for the presence of genomic variants that have been associated in the literature with other phenotypes present in the proband, primarily obesity and nephropathy. A summary of the relevant findings is shown in Table 3. 


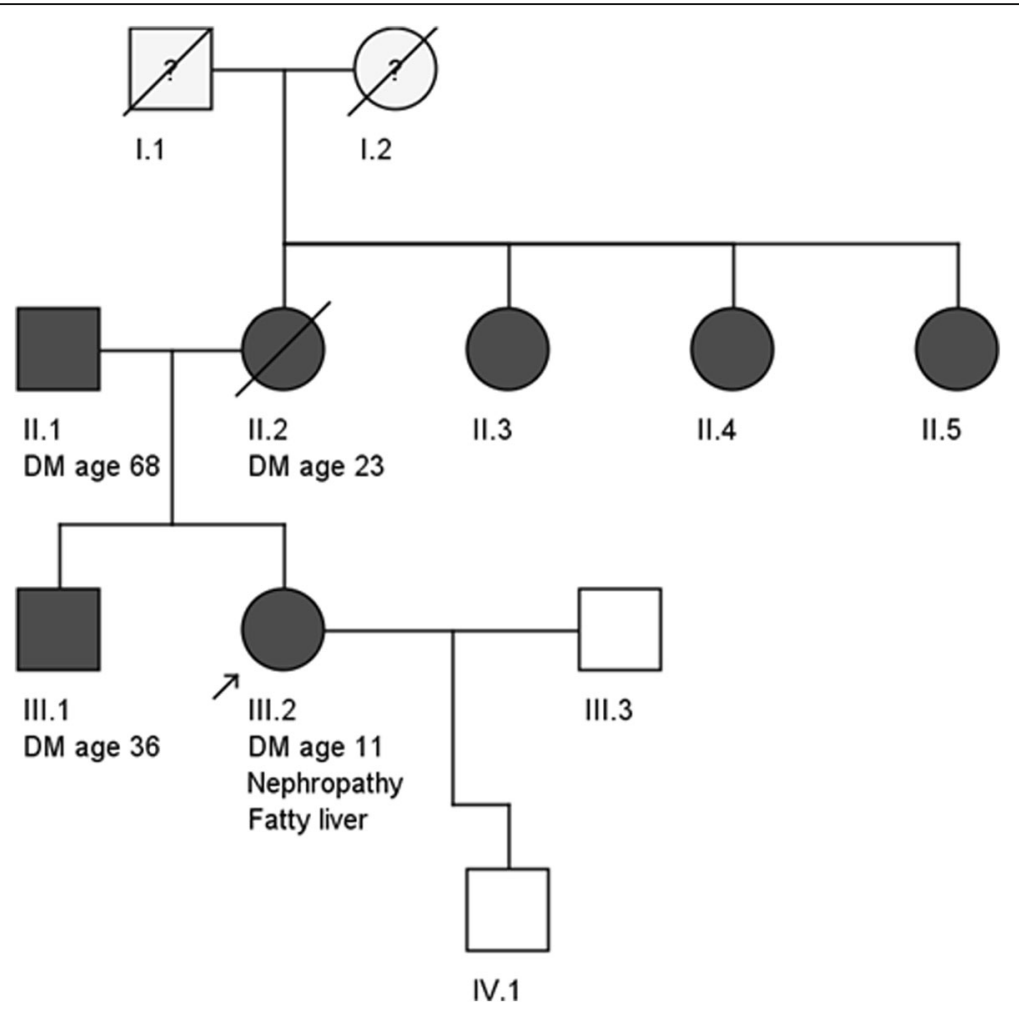

Fig. 1 Pedigree of the affected family

\section{Discussion}

In the present report, whole exome sequencing revealed a rare missense mutation in HNF1 $\beta$ that shows high conservation scores and has in silico predictions in favour of pathogenicity. This mutation is located in the C-terminal transactivation domain of HNF1 $\beta$, is absent from controls and is present at an extremely low frequency in the Exome Aggregation Consortium dataset. Furthermore, the proband's uncharacteristic clinical phenotype and family history is suggestive of atypical or monogenic diabetes, although further evaluation of the mutation is required to provide a conclusive diagnosis of MODY5 in this case. Multiple prediction algorithms demonstrate a deleterious effect of this mutation on the

Table 1 Whole exome sequencing detail of coverage and number of read

\begin{tabular}{ll}
\hline Number of reads in raw sequence & $55,723,934$ \\
Number of reads after de-duplication & $96.63 \%$ \\
Number of mapped reads & $99.53 \%$ \\
Number of mapped reads to targeted & $79.11 \%$ \\
regions of the genome & \\
Average depth & $66.72 \%$ \\
Coverage 10x & $98.67 \%$ \\
Coverage 20x & $93.93 \%$ \\
Coverage 50x & $58.10 \%$ \\
\hline
\end{tabular}

gene. Missense mutations in HNF1 $\beta$ are commonly associated with both monogenic diabetes and kidney disease, and a number of in-vitro functional studies have demonstrated that missense mutations in HNF1 $\beta$ lead to impaired DNA-binding and reduced transactivation potential [23-25]. In view of these criteria, the mutation can be classified as likely pathogenic according to the established guidelines from the American College of Medical Genetics and Genomics and the Association for Molecular Pathology (ACMG/AMP) (criteria PP2, PP2, PP4, PM1, PM2) [26].

To date, around 230 mutations in HNF1 $\beta$ have been described [27]. This gene belongs to the homeodomaincontaining family of transcription factors and is involved in the organogenesis of the kidneys, urinary tract, liver and pancreas. HNF1 $\beta$ functions as a homo- or heterodimer with a structurally related transcription factor HNF1 $\alpha$ [28]. Mutations in HNF1 $\alpha$ are responsible for the commonest type of monogenic diabetes (MODY3), characterised by progressive glucose intolerance due to an insulin secretory defect [7]. In contrast, mutations in HNF1 $\beta$ are associated with a wide array of clinical phenotypes that can include renal disease, and which are distinguished by the absence of clear genotype-phenotype associations. Primarily, heterozygous mutations in HNF1 $\beta$ cause a complex Renal cysts and Diabetes syndrome (RCAD) characterised by early onset diabetes (MODY5), 
Table 2 In-silico predictors of pathogenicity and evolutionary conservation analysis for the HNF1 $\beta$ mutation described in the text In-silico prediction of pathogenicity for HNF1 $\beta$ c.1580G > A (p.Arg527GIn)

\begin{tabular}{lll} 
Tool & Prediction & Score \\
MutationTaster & Disease causing & \\
MutationAssessor & Medium & 2.48 \\
FATHMM-MKL & Damaging & 0.9625 \\
MetaSVM & Damaging & 0.9934 \\
MetalR & Damaging & 0.9388 \\
LRT & Deletrious & 0 \\
PolyPhen-2 - HumVar & Probably damaging & 0.998 \\
SIFT & Damaging & 0.038 \\
DAMN score & 0.9996 & \\
CADD scaled score & & 35 \\
Evolutionary conservation analysis for HNF1 $\beta$ c.1580G > A (p.Arg527Gln) \\
Genomic Evolutionary Rate Profiling (GERP) & 5.63 \\
PhyloP20way - mammalian & & 0.935 \\
SiPhy29way - mammalian & & 0.9297 \\
\hline
\end{tabular}

liver dysfunction and pancreatic hypoplasia. The extent and severity of renal disease varies extensively in HNF1 $\beta$ mutations, ranging from congenital anomalies of the kidney and urinary tract (CAKUT), cystic kidneys to hyperuricaemia [29-31]. HNF1 $\beta$ mutations have been associated with isolated renal disease in the absence of diabetes and conversely, HNF1 $\beta$ deletions with youngonset diabetes but no kidney disease have also been described [32, 33]. Compared to other MODY genes, a high rate of de-novo mutations in HNF1 $\beta$ has been reported in the literature [29]. The variable expressivity of HNF1 $\beta$ mutations also extends to the type and age of onset of renal disease, which ranges from intrauterine life to middle age [34]. A recent multicentre retrospective cohort study of patients with HNF1 $\beta$ mutations showed that stage 3-4 chronic kidney disease was present in $44 \%$ of cases, and end-stage renal disease in $21 \%$ of cases [35].

The diabetic phenotype associated with HNF1 $\beta$ mutations is also equally heterogenous, with severity of glycaemia ranging from impaired glucose tolerance to diabetes requiring insulin therapy. The mean age of diagnosis of diabetes is 26 years, with a range of 10-61 years [36]. HNF1 $\beta$ mutations

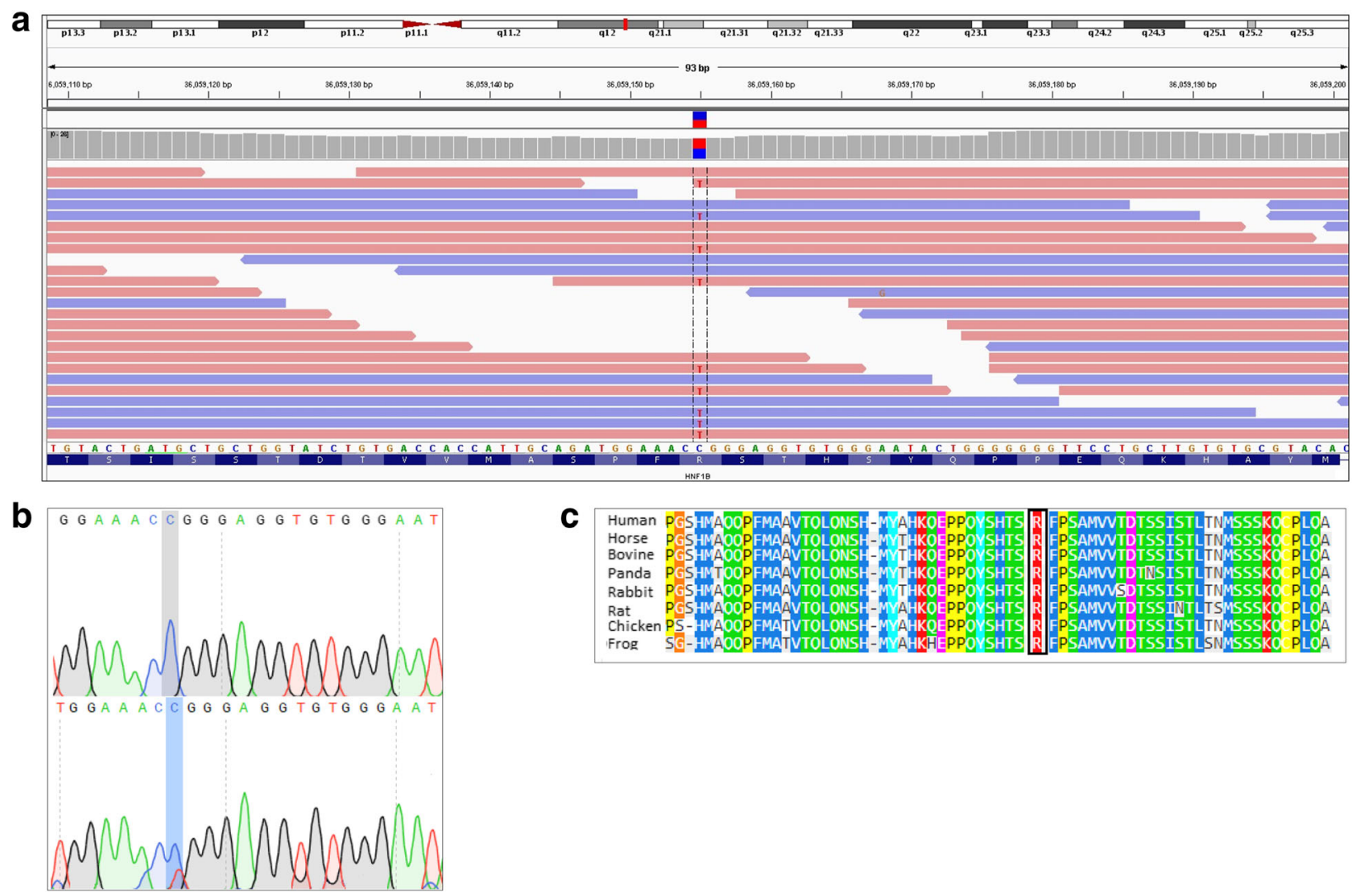

Fig. 2 a: Excerpt of exome sequencing data visualised with Integrative Genomics viewer. The heterozygous C > T mutation at chromosome 17, position 36,059,155, in exon 8 of HNF1 $\beta$ is shown in the reverse strand. b: Sanger sequencing trace showing normal [top] and mutated [bottom] sequence. c: Multiple sequence alignment showing 66 amino acids surrounding the mutation position, indicated by the black box. The amino acid residue affected is highly conserved across multiple species 
Table 3 List of genomic variants and their respective minor allele frequency (1000 Genomes, TSI dataset) that have been identified in the proband

\begin{tabular}{|c|c|c|c|c|c|c|c|c|}
\hline Chromosome & Position & dbSNP & Variant & Type & Gene & Effect & MAF & Clinical significance \\
\hline chr3 & $10,331,457$ & rs696217 & $\mathrm{G} / \mathrm{T}$ & Missense & GHRL & p.Leu60Met & 0.07 & Risk factor for metabolic syndrome and childhood obesity \\
\hline chr6 & $132,172,368$ & rs1044498 & $\mathrm{A} / \mathrm{C}$ & Missense & ENPP1 & p.Lys173Gln & 0.13 & Susceptibility to Insulin resistance \\
\hline chr4 & $6,292,915$ & rs10010131 & $A / G$ & Intronic & WFS1 & & 0.36 & Type 2 diabetes risk \\
\hline chr6 & $149,721,690$ & rs237025 & $\mathrm{G} / \mathrm{A}$ & Missense & SUMO4 & p.Val55Met & 0.45 & Type 2 diabetes risk, diabetic nephropathy \\
\hline chr13 & $110,435,231$ & rs1805097 & $C / T$ & Missense & IRS2 & p.Gly1057Asp & 0.33 & Type 2 diabetes risk \\
\hline chr11 & $17,409,572$ & rs5219 & $\mathrm{T} / \mathrm{C}$ & Missense & KCNJ11 & p.Lys23Glu & 0.29 & Type 2 diabetes risk \\
\hline chr6 & $160,113,872$ & rs4880 & $A / G$ & Missense & SOD2 & p.Val16Ala & 0.47 & Diabetic nephropathy \\
\hline chr2 & $25,141,538$ & rs11676272 & $A / G$ & Missense & $\mathrm{ADCY} 3$ & p.Ser107Pro & 0.42 & Childhood obesity \\
\hline
\end{tabular}

The variants in this table have been associated with obesity, type 2 diabetes and nephropathy in different studies

lead to beta cell dysfunction and reduced insulin secretion, which may be associated with pancreatic hypoplasia [37].

The exact cause of albuminuria and hyperfiltration in our proband is difficult to ascertain, as no evidence of renal cystic disease or of CAKUT was present on imaging. While renal cystic disease is a major manifestation of HNF1 $\beta$ mutations ( $65 \%$ of cases), considerable variation in renal phenotype exists. Similarly, studies have also shown that when renal biopsies are performed there is heterogeneity in the histological diagnosis [36]. Despite the presence of diabetes, the renal dysfunction found in carriers of HNF $1 \beta$ mutations is thought to be caused by renal development abnormalities rather than diabetic renal disease [38]. However, the proband also had microvascular complications of diabetes and poor glycaemic control, making associated diabetic nephropathy highly probable. A recent case report has described histologically-proven diabetic kidney disease (DKD) with glomerular sclerosis, severe diffuse mesangial cell proliferation, matrix expansion and arteriole hyalinosis in a 30 year old female with MODY 5 and macroalbuminuria caused by a missense mutation in exon 4 of HNF1 $\beta$ [39]. While glomerular hyperfiltration associated with early onset DM is a powerful risk factor for the development of progressive diabetic nephropathy, it is more likely attributed to glomerular DKD in our proband. Interestingly, another case report has described end-stage renal failure secondary to probable DKD in a patient with HNF1 $\beta$ mutation and RCAD syndrome [40].

We also examined exome data, focusing on susceptibility genes for early onset obesity, diabetic kidney disease and type 2 diabetes mellitus. Several common susceptibility variants were detected. Presumably, the additive effects of common risk variants contributes to the presentation, clinical course and progression of diabetes in the proband.

This report is significant for a number of reasons. Primarily, it expands on the mutational spectrum and wide array of clinical phenotypes associated with the HNF1 $\beta$ gene. In the era of personalised medicine, it is increasingly important to place the clinical phenotype into a genespecific context that would enable clinicians to determine both immediate and future clinical implications to both the patient and family members. Secondly, it adds to the few clinical reports that highlight the presence of earlystage diabetic nephropathy with hyperfiltration due to probable glomerular DKD in the absence of renal cysts or renal morphological abnormalities in MODY5. Furthermore, it is important to emphasise that clinicians should maintain a high index of suspicion for monogenic diabetes in young onset non-autoimmune disease, and should consider referral for HNF1 $\beta$ mutation analysis in the presence of renal cysts with or without diabetes. The presence of obesity from a young age in the proband is also significant, in view of the fact that the initial diagnosis was of earlyonset type 2 diabetes rather than monogenic diabetes. The report also reinforces the need for increased research on the molecular epidemiology of monogenic diabetes in the Maltese islands. As yet, the prevalence of MODY in Malta is not determined. It is likely to show significant differences from studies carried out in northern European populations, in part due to strong founder effects that have been reported on the island [41]. Of note, the same missense mutation in HNF1 $\beta$ has also been detected in an unrelated Maltese female referred for monogenic diabetes screening. The second case involves a 30 year old female with diabetes since age 13, a family history of paternallytransmitted early-onset diabetes, a BMI of $26.32 \mathrm{~kg} /$ $\mathrm{m}^{2}$, no history of diabetic ketoacidosis and treatment with long-acting insulin. Similarly, no renal cysts or structural renal tract anomalies were identified but investigation showed microalbuminuria from longstanding diabetes. The identification of the same missense mutation in two unrelated Maltese probands both with early-onset diabetes strongly suggests a possible genetic founder effect for this mutation. The authors are presently expanding genetic epidemiology studies to further investigate this. 


\section{Conclusion}

In conclusion, this report has described a rare missense mutation in exon 8 of HNF1 $\beta$ with multiple in-silico predictions consistent with pathogenicity. The proband had childhood onset atypical diabetes complicated by obesity and early stage diabetic nephropathy in the absence of renal cysts. The broad phenotypic variability of both diabetic and renal disease probably accounts for underdiagnoses of HNF1 $\beta$ mutations in clinical practice. The authors also emphasise that clinicians should be vigilant for the possibility of monogenic diabetes even in obese patients with a strong family history and a high probability on MODY risk calculators. This is particularly relevant in specific populations such as in the Maltese islands, where the population carries high prevalence rates for both obesity and diabetes, and the genetic epidemiology of the disease is as yet uncharacterised.

\section{Abbreviations}

CAKUT: Congenital anomalies of the kidney and urinary tract; DKD: Diabetic kidney disease; HNF: Hepatocyte nuclear factor; MODY: Maturity onset diabetes of the young; RCAD: Renal cysts and diabetes; SNP: Single nucleotide polymorphism

\section{Acknowledgements}

We thank the proband for her collaboration in this study.

\section{Funding}

This work was supported by institutional funds of the University of Malta.

\section{Availability of data and materials}

The dataset analysed during the current study are available from the corresponding author on reasonable request.

\section{Authors' contributions}

NPP and JC: Data acquisition, sequencing, analysis and clinical interpretation of data, drafting of the manuscript. JV and AF: study concept and design, analysis and interpretation of data, drafting and critical revision of the manuscript. All the authors read and approved the final manuscript.

\section{Ethics approval and consent to participate}

The study was approved by the Ethical Committee of the University of Malta and the patient gave written informed consent to participate. This study was performed according to Declaration of Helsinki.

\section{Consent for publication}

Written informed consent was obtained from the proband for the publication of this case report.

\section{Competing interests}

The authors declare that they have no competing interests.

\section{Publisher's Note}

Springer Nature remains neutral with regard to jurisdictional claims in published maps and institutional affiliations.

\section{Author details}

${ }^{1}$ Centre for Molecular Medicine and Biobanking, University of Malta, Msida, Malta. ${ }^{2}$ Department of Obstetrics and Gynaecology, University of Malta, Msida, Malta. ${ }^{3}$ Department of Medicine, University of Malta, Msida, Malta.
Received: 17 January 2018 Accepted: 3 May 2018

Published online: 15 May 2018

\section{References}

1. Fendler W, Borowiec M, Baranowska-Jazwiecka A, Szadkowska A, SkalaZamorowska E, Deja G, et al. Prevalence of monogenic diabetes amongst polish children after a nationwide genetic screening campaign. Diabetologia. 2012;55:2631-5.

2. Pihoker C, Gilliam LK, Ellard S, Dabelea D, Davis C, Dolan LM, et al. Prevalence, characteristics and clinical diagnosis of maturity onset diabetes of the young due to mutations in HNF1A, HNF4A, and Glucokinase: results from the SEARCH for diabetes in youth. J Clin Endocrinol Metab. 2013;98:4055-62.

3. Shepherd M, Shields B, Hammersley S, Hudson M, McDonald TJ, Colclough $\mathrm{K}$, et al. Systematic population screening, using biomarkers and genetic testing, identifies $2.5 \%$ of the UK pediatric diabetes population with monogenic diabetes. Diabetes Care. 2016;39:1879-88.

4. Misra S, Shields B, Colclough K, Johnston DG, Oliver NS, Ellard S, et al. South Asian individuals with diabetes who are referred for MODY testing in the UK have a lower mutation pick-up rate than white European people. Diabetologia. 2016:59:2262-5.

5. Kanthimathi S, Jahnavi S, Balamurugan K, Ranjani H, Sonya J, Goswami S, et al. Glucokinase gene mutations (MODY 2) in Asian Indians. Diabetes Technol Ther. 2014;16:180-5.

6. Rubio-Cabezas O, Hattersley AT, Njølstad PR, Mlynarski W, Ellard S, White N, et al. ISPAD clinical practice consensus guidelines 2014. The diagnosis and management of monogenic diabetes in children and adolescents. Pediatr Diabetes. 2014;15(Suppl 20):47-64.

7. Shields BM, Hicks S, Shepherd MH, Colclough K, Hattersley AT, Ellard S. Maturity-onset diabetes of the young (MODY): how many cases are we missing? Diabetologia. 2010;53:2504-8.

8. Shields BM, McDonald TJ, Ellard S, Campbell MJ, Hyde C, Hattersley AT. The development and validation of a clinical prediction model to determine the probability of MODY in patients with young-onset diabetes. Diabetologia. 2012;55:1265-72.

9. Li H, Durbin R. Fast and accurate short read alignment with burrowswheeler transform. Bioinformatics. 2009;25:1754-60.

10. Picard. http://broadinstitute.github.io/picard. Accessed 7 May 2018

11. Cock PJA, Fields CJ, Goto N, Heuer ML, Rice PM. The sanger FASTQ file format for sequences with quality scores, and the Solexa/Illumina FASTQ variants. Nucleic Acids Res. 2010;38:1767-71.

12. McKenna A, Hanna M, Banks E, Sivachenko A, Cibulskis K, Kernytsky A, et al. The genome analysis toolkit: a MapReduce framework for analyzing next-generation DNA sequencing data. Genome Res. 2010;20:1297-303.

13. Li H, Handsaker B, Wysoker A, Fennell T, Ruan J, Homer N, et al. The sequence alignment/map format and SAMtools. Bioinformatics. 2009;25:2078-9.

14. Kumar P, Henikoff S, Ng PC. Predicting the effects of coding nonsynonymous variants on protein function using the SIFT algorithm. Nat Protoc. 2009;4:1073-81.

15. Adzhubei IA, Schmidt S, Peshkin L, Ramensky VE, Gerasimova A, Bork P, et al. A method and server for predicting damaging missense mutations. Nat Methods. 2010;7:248-9.

16. Dong C, Wei $P$, Jian X, Gibbs R, Boerwinkle E, Wang $K$, et al. Comparison and integration of deleteriousness prediction methods for nonsynonymous SNVs in whole exome sequencing studies. Hum Mol Genet. 2015:24:2125-37.

17. Shihab HA, Rogers MF, Gough J, Mort M, Cooper DN, Day INM, et al. An integrative approach to predicting the functional effects of non-coding and coding sequence variation. Bioinformatics. 2015;31:1536-43.

18. Quang D, Chen $Y$, Xie X. DANN: a deep learning approach for annotating the pathogenicity of genetic variants. Bioinformatics. 2015;31:761-3.

19. Kircher M, Witten DM, Jain P, O'Roak BJ, Cooper GM, Shendure J. A general framework for estimating the relative pathogenicity of human genetic variants. Nat Genet. 2014;46:310-5.

20. Schwarz JM, Cooper DN, Schuelke M, Seelow D. MutationTaster2: mutation prediction for the deep-sequencing age. Nat Methods. 2014;11:361-2.

21. Reva B, Antipin Y, Sander C. Determinants of protein function revealed by combinatorial entropy optimization. Genome Biol. 2007;8:R232.

22. Chun S, Fay JC. Identification of deleterious mutations within three human genomes. Genome Res. 2009;19:1553-61. 
23. Kim EK, Lee JS, Cheong HI, Chung SS, Kwak SH, Park KS. Identification and functional characterization of P159L mutation in HNF1B in a family with maturity-onset diabetes of the young 5 (MODY5). Genomics Inform. 2014;12:240-6.

24. Barbacci E, Chalkiadaki A, Masdeu C, Haumaitre C, Lokmane L, Loirat C, et al. HNF1beta/TCF2 mutations impair transactivation potential through altered co-regulator recruitment. Hum Mol Genet. 2004;13:3139-49.

25. Raaijmakers A, Corveleyn A, Devriendt K, Tienoven V, Pieter T, Allegaert K, et al. Criteria for HNF1B analysis in patients with congenital abnormalities of kidney and urinary tract. Nephrol Dial Transplant. 2015;30:835-42.

26. Richards S, Aziz N, Bale S, Bick D, Das S, Gastier-Foster J, et al. Standards and guidelines for the interpretation of sequence variants: a joint consensus recommendation of the American College of Medical Genetics and Genomics and the Association for Molecular Pathology. Genet med off J am Coll. Med Genet. 2015:17:405-24.

27. Stenson PD, Mort M, Ball EV, Evans K, Hayden M, Heywood S, et al. The human gene mutation database: towards a comprehensive repository of inherited mutation data for medical research, genetic diagnosis and nextgeneration sequencing studies. Hum Genet. 2017;136:665-77.

28. Mendel DB, Hansen LP, Graves MK, Conley PB, Crabtree GR. HNF-1 alpha and HNF-1 beta (VHNF-1) share dimerization and homeo domains, but not activation domains, and form heterodimers in vitro. Genes Dev. 1991;5:1042-56.

29. Bellanné-Chantelot C, Chauveau D, Gautier J-F, Dubois-Laforgue D, Clauin S, Beaufils $\mathrm{S}$, et al. Clinical spectrum associated with hepatocyte nuclear factor1 beta mutations. Ann Intern Med. 2004;140:510-7.

30. Raile K, Klopocki E, Holder M, Wessel T, Galler A, Deiss D, et al. Expanded clinical spectrum in hepatocyte nuclear factor $1 \mathrm{~b}$-maturity-onset diabetes of the young. J Clin Endocrinol Metab. 2009;94:2658-64.

31. Bockenhauer D, Jaureguiberry G. HNF1B-associated clinical phenotypes: the kidney and beyond. Pediatr Nephrol. 2016;31:707-14.

32. Weber S, Moriniere V, Knüppel T, Charbit M, Dusek J, Ghiggeri GM, et al. Prevalence of mutations in renal developmental genes in children with renal hypodysplasia: results of the ESCAPE study. J Am Soc Nephrol. 2006;17:2864-70

33. Edghill EL, Stals K, Oram RA, Shepherd MH, Hattersley AT, Ellard S. HNF1B deletions in patients with young-onset diabetes but no known renal disease. Diabet Med J Br Diabet Assoc. 2013;30:114-7.

34. Heidet L, Decramer S, Pawtowski A, Morinière V, Bandin F, Knebelmann B, et al. Spectrum of HNF1B mutations in a large cohort of patients who harbor renal diseases. Clin J Am Soc Nephrol. 2010;5:1079-90.

35. Dubois-Laforgue D, Cornu E, Saint-Martin C, Coste J, Bellanné-Chantelot C, Timsit J, et al. Diabetes, associated clinical Spectrum, long-term prognosis and genotype/phenotype correlations in 201 adult patients with hepatocyte nuclear factor 1 B (HNF1B) molecular defects. Diabetes Care. 2017:40:1436-43

36. Bingham C, Hattersley AT. Renal cysts and diabetes syndrome resulting from mutations in hepatocyte nuclear factor-1 13 . Nephrol Dial Transplant. 2004;19:2703-8

37. Gautier J, Bellanne-chantelot C, Dubois-laforgue D, Wilhelm J, Boitard C, Clauin $\mathrm{S}$, et al. Multi-organ damage in Mody 5 related to mutations of the hepatocyte nuclear factor-1ßgene. Diabetes. 2002;51 https://insights.ovid. com/diabetes/diab/2002/06/002/multi-organ-damage-mody5-relatedmutations/1049/00003439. Accessed 9 May 2018.

38. Magee GM, Bilous RW, Cardwell CR, Hunter SJ, Kee F, Fogarty DG. Is hyperfiltration associated with the future risk of developing diabetic nephropathy? A meta-analysis. Diabetologia. 2009;52:691-7.

39. Wang $Y$, Zhao $Y$, Zhang J, Yang Y, Liu F. A case of a novel mutation in HNF1 $\beta$-related maturity-onset diabetes of the young type 5 with diabetic kidney disease complication in a Chinese family. J Diabetes Complicat. 2017;31:1243-6.

40. Hegde P, Meldon A, Lamen L, Sharma D, Kalathil D. An interesting unfolding of the diagnosis of hepatocyte nuclear factor-1 beta (HNF1 $\beta$ ) monogenic diabetes. Pract Diabetes. 2017;34:320-322a.

41. Farrugia R, Scerri CA, Montalto SA, Parascandolo R, Neville BGR, Felice AE. Molecular genetics of tetrahydrobiopterin (BH4) deficiency in the Maltese population. Mol Genet Metab. 2007;90:277-83.

\section{Ready to submit your research? Choose BMC and benefit from:}

- fast, convenient online submission

- thorough peer review by experienced researchers in your field

- rapid publication on acceptance

- support for research data, including large and complex data types

- gold Open Access which fosters wider collaboration and increased citations

- maximum visibility for your research: over $100 \mathrm{M}$ website views per year

At BMC, research is always in progress.

Learn more biomedcentral.com/submissions 